\title{
Producción de contenidos digitales, una oportunidad para los profesionales de las TIC
}

\author{
Digital contents production, an opportunity for the \\ profesionals in information and communication technologies
}

CLARA PATRICIA AVELLA IBÁÑNZ

Ingeniera de Sistemas

MSc. Ingeniería de Sistemas y Computación

Grupo de Investigación EDUTIC

Universidad de Boyacá, Colombia

cpavella@uniboyaca.edu.co

ÉRIKA MARÍA SANDOVAL VALERO

Ingeniera de Sistemas

Esp. Gerencia de Sistemas Informáticos

Grupo de Investigación EDUTIC

Universidad de Boyacá, Colombia

erisandoval@uniboyaca.edu.co

CARMENZA MONTAÑEZ TORRES

Ingeniera de Sistemas

Mg. Dirección Estratégica en TIC

Grupo de Investigación EDUTIC

Universidad de Boyacá, Colombia

carmenzamt@uniboyaca.edu.co

Recibido: 04/05/2012

Aceptado: 05/10/2012 


\section{RESUMEN}

Este escrito de reflexión es producto del análisis al estado del arte del proyecto denominado "Integración de estrategias y metodologías para la producción y evaluación de contenidos digitales educativos", desarrollado por los autores, integrantes del grupo de investigación EDUTIC de la Universidad de Boyacá.

La industria de contenidos digitales incluye productos y tecnologías relacionadas con la animación digital, el e-learning, los libros digitales, los contenidos informativos que circulan por la web, la música digital, la televisión y la radio digital, los portales online y los juegos online; es decir, se trata de los servicios que se ofrecen, se transmiten y se almacenan a través de las nuevas tecnologías de la información y las comunicaciones.

Al analizar el estado del arte de la investigación mencionada, se identificó una gran oportunidad de desarrollo para diversas disciplinas profesionales, destacándose entre ellas las TIC (Tecnologías de la Información y las Comunicaciones). Esta oportunidad abre espacios en los siguientes ámbitos: generación de nuevas empresas, diseño e innovación, gestión y producción de contenidos digitales, así como la creación de nuevos programas de formación académica en los mismos. El camino está dado para aprovechar las políticas que el gobierno colombiano ha generado a través de sus Ministerios de TIC y de Educación, para incentivar y poner en marcha nuevos proyectos en el sector de la industria creativa.

Este artículo presenta algunas oportunidades que surgen en torno a la industria de la producción de contenidos digitales y en las cuales pueden tener participación los profesionales de áreas relacionadas con las TIC.

Palabras clave: Contenidos digitales, economía creativa, emprendimiento, industrias creativas. TIC

\section{ABSTRACT}

This brief reflection is a product of the analysis to the state of the art of the project named "Integration of strategies and methodologies for the production and evaluation of educational digital content", developed by the authors, members of the research group EDUTIC of the University of Boyacá.

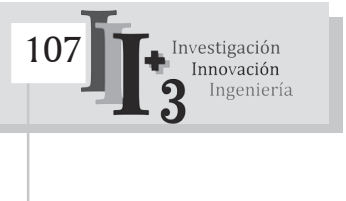


The digital content industry includes products and technologies related to digital animation, e-learning, digital books, information content circulating on the web, digital music, television and digital radio, online portals and games; that means, the services that are offered, transmitted and stored by the new information technologies and communications technologies.

After analyzing the state of the art of the project mentioned above, a great development opportunity for professional in different disciplines was identified, among them, the professionals in information and communications technologies. This opportunity opens up spaces in the following areas: proposal of new business, design and innovation, management and digital content production, and the creation of new academic programs in the same areas. The way is given to take advantage of policies that the Colombian Government has created through its Ministry of Information and communications Technologies and Ministry of Education, in order to encourage and implement new projects in the creative industry sector.

This article presents some opportunities that arise around digital content production industry and on which ICT professionals might participate.

Keywords: Digital contents, creative economy, venture, creative industries, ICT

\section{INTRODUCCIÓN}

El propósito de este escrito es conocer el proceso que ha tenido la producción de contenidos digitales, puesto que en estos momentos es un nicho de mercado mundial, cuyo crecimiento se está acelerando con el paso del tiempo. Las Naciones Unidas en su reporte sobre Economía Creativa (Orduz 2010), promocionan e invitan a la comunidad mundial para que definan estrategias y políticas encaminadas al desarrollo de esta industria.

En la política de promoción de la industria de contenidos digitales, publicado por el Ministerio de Tecnologías de la información y las comunicaciones TIC, se señala que en Colombia, la industria de producción de contenidos digitales tiene una poca participación, razón por la cual el gobierno a través del Ministerio de TIC se ha propuesto impulsar esta industria, teniendo en cuenta que ofrece grandes oportunidades de crecimiento y desarrollo para el país. La primera acción que el Ministerio de TIC ha iniciado, es la creación de una política pública de contenidos digitales (denominada Vive Digital), la cual se está construyendo actualmente con el aporte de los colombianos. El gobierno Colombiano está buscando que esta política vaya a tono con la tendencia mundial en esta industria, definiendo acciones para incentivar su crecimiento. 
Este artículo está organizado de la siguiente manera: El primer apartado presenta el desarrollo que ha tenido la industria de contenidos digitales en el mundo durante los últimos años; luego se describe el fomento que se le ha dado a la misma. Los avances respecto a la producción de contenidos digitales en Colombia se exponen en seguida, finalizando con el apartado en el cual se revelan algunas oportunidades concretas para generar empresa y nuevas alternativas de capacitación en el sector de los contenidos digitales.

\section{DESARROLLO MUNDIAL DE LA INDUSTRIA DE CONTENIDOS DIGITALES}

Los contenidos digitales son un enorme mercado de crecimiento mundial, teniendo en cuenta que este crecimiento se ha acelerado en los últimos cinco años y es exponencial para los próximos 15 años. (Dinero.com, 2010).

La industria de contenidos digitales ha tenido un desarrollo ascendente en los últimos años, especialmente en Canadá y Estados Unidos, seguidos por Europa y Asia. En el caso de Iberoamérica se destaca el crecimiento de esta industria en España, seguido por países como México, Brasil, Argentina y Colombia. Algunas estadísticas presentadas en el primer Foro de Contenidos Digitales (Restrepo, 2011), que se presentan a continuación, dan cuenta del desarrollo de la misma industria de contenidos digitales.

\section{Aplicaciones móviles}

- El mercado de aplicaciones móviles ha subido de 0 en el año 2004 a 24 millones de descargas diarias en 2011.

- Apple ha pagado más de 2.000 millones de dólares a programadores de aplicaciones móviles.

\section{Video-juegos}

- El mercado de video juegos online y móviles crecerá de 19.000 millones de dólares en 2009 a 44.000 millones en 2014 .

- Los juegos sociales crecieron de cero jugadores en 2005 a 300 millones en 2011.

- Sólo en Facebook, los juegos generan 15 millones de dólares diarios.

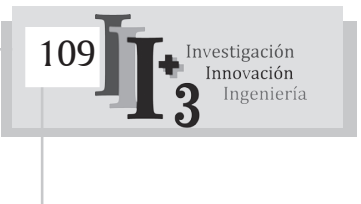




\section{Animación digital}

- La producción global de animación digital alcanzó 177.000 millones de dólares en 2009 y se proyectó que alcanzaría los 249.000 millones en 2012.

- La mayoría de los segmentos de animación digital crecen en promedio 10\% anual. La distribución entre las diferentes regiones del mundo se puede ver en la figura 1.

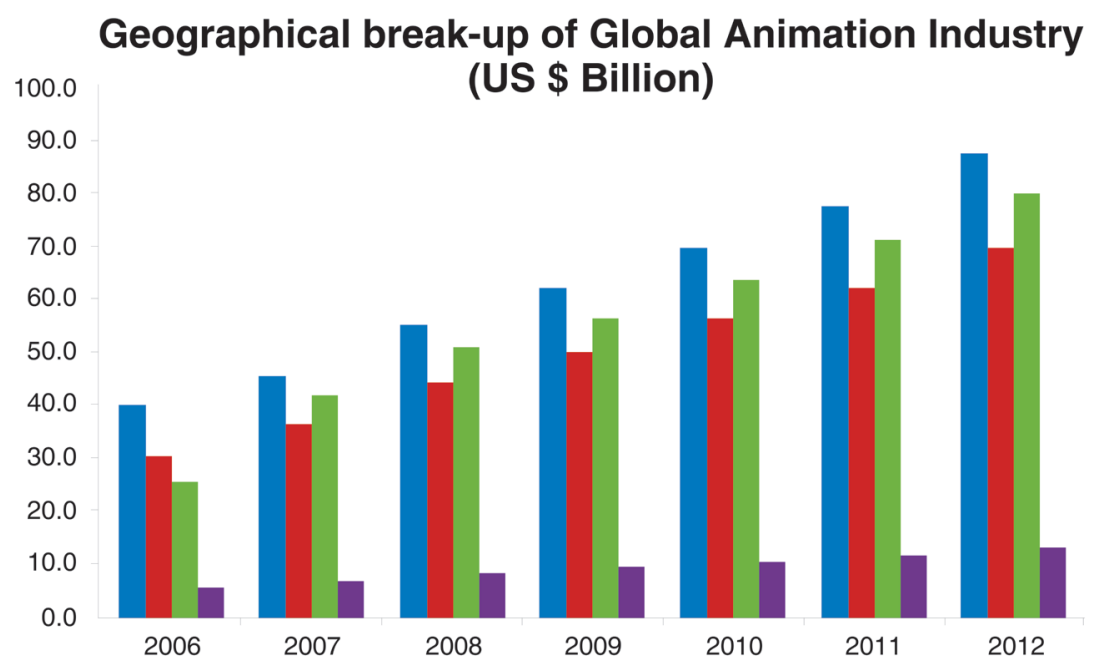

Figura 1. Distribución geográfica de la industria de animación (Billones de dólares). Fuente: Restrepo, 2011.

\section{Publicidad digital}

- Se proyecta que el mercado mundial de publicidad digital en 2014 será de 97.000 millones de dólares.

- El mercado de la publicidad digital en Colombia alcanzó los 47.000 millones de pesos en el año 2010 (crecimiento de 55.77\% frente al año 2009).

\section{Video en línea}

- Cada día se venden aproximadamente 1.200 millones de videos en YouTube.

- 86\% de los usuarios de Internet de Estados Unidos vieron videos en línea en el año 2010. 


\section{Libros digitales}

- En el Reino Unido, en 2010 el mercado de los libros digitales creció en un 300\%.

\section{Bienes virtuales}

Se estima que en el año 2010 el mercado mundial de bienes virtuales fue de 7.300 millones de dólares y en 2014 este mercado aumentará a más de 14.000 millones de dólares.

\section{Exportaciones de contenidos digitales}

En América Latina y el Caribe las exportaciones de bienes creativos casi se duplicaron, pasando de aproximadamente 5.500 a 9.000 mil millones de dólares. El nivel de las exportaciones de la región sigue siendo relativamente bajo, dado el potencial de sus industrias creativas. Sólo México figura entre los 20 principales exportadores. Otros exportadores de la región son Brasil, Colombia y Argentina. (UNCTAD, 2010).

\section{Participación de Latinoamérica en la industria de contenidos digitales}

Actualmente, América Latina participa en la industria mundial de contenidos digitales, sólo con el 1.9\%, esto es lo que lleva al reto de promover esta industria en nuestro país. (Dinero.com, 2010).

\section{FOMENTO A LA INDUSTRIA DE CONTENIDOS DIGITALES EN EL MUNDO}

La UNCTAD - United Nations Conference on Trade and Development (2010) revela que la industria de contenidos digitales tiene un gran potencial para el desarrollo de los países que buscan diversificar sus economías y participar en uno de los sectores más dinámicos de la economía mundial. Los siguientes párrafos presentan algunos aspectos claves analizados por la UNCTAD para promocionar la industria de contenidos digitales.

Para los países que desean desarrollar la industria de contenidos digitales, el punto de partida debe ser el mejoramiento de sus capacidades creativas y la identificación de los sectores creativos con mayor potencial. Para esto es necesario definir políticas transversales articuladas. Los esfuerzos de estos países deberían estar orientados hacia la funcionalidad de "nexos creativos" capaces de atraer inversionistas, construir capacidades empresariales creativas y ofrecer mejor acceso e infraestructura a TIC modernas. Esto lleva a que los países se beneficien de la convergencia digital global y se optimice el comercio potencial de los productos creativos, en los mercados domésticos e internacionales. 
De otra parte, las estrategias para fomentar el desarrollo de la economía creativa deben reconocer la naturaleza multidisciplinaria de esta industria, es decir, su vínculo con los sectores económico, social, cultural, tecnológico y medioambiental. También es muy importante definir una reglamentación para proteger los derechos de autor y la propiedad intelectual. La economía creativa, traspasa las artes, los negocios y la conectividad e impulsa la innovación y los nuevos modelos de negocio. La era digital facilita el mercadeo y la distribución de canales para música, animación digital, películas, noticias, publicidad, entre otras.

El desarrollo de la industria de dispositivos móviles ha sido un fenómeno potente para el desarrollo de todos los países del mundo, sin embargo, hay muchos países en vía de desarrollo que están rezagados en términos de ancho de banda en conectividad. Para las industrias creativas, esto ha sido una restricción, porque muchas aplicaciones para estimular la producción creativa y los negocios electrónicos requieren de un ancho de banda suficientemente amplio. Esto lleva a que los esfuerzos de investigación en el ámbito nacional y regional deban ser guiados, en colaboración con agencias internacionales, con el fin de buscar una mejor infraestructura para contar con un buen ancho de banda.

Las políticas para la economía creativa han respondido; no sólo a las necesidades económicas, sino también a las solicitudes especiales de las comunidades locales relacionadas con la educación, la identidad cultural, la inequidad social y los problemas del medio ambiente. Un gran número de poblaciones a nivel mundial han usado el concepto de ciudades creativas para formular estrategias de desarrollo urbano, con el fin de fortalecer el crecimiento regional con un enfoque en la cultura y las actividades creativas.

Cada país es diferente, cada mercado es especial y cada producto creativo tiene su toque y realce específico. Sin embargo, cada país puede ser capaz de identificar sus industrias creativas claves, que no hayan sido explotadas a su máximo potencial para obtener grandes beneficios. Cada país debería formular una estrategia para fomentar su economía creativa, basado en sus propias fortalezas, debilidades y realidades, y este es el momento para hacerlo.

\section{LA INDUSTRIA DE CONTENIDOS DIGITALES EN COLOMBIA}

A continuación se presentan los avances que ha tenido la producción de contenidos digitales en Colombia, algunas estadísticas relacionadas con el tema y las políticas del gobierno nacional para incentivar esta industria.

\section{Avances respecto a la producción de contenidos digitales en Colombia}

En la última década, la Organización de Naciones Unidas, a través del Creative Economy Report (UNCTAD, 2010) presenta estudios realizados en el sector de contenidos digitales, con el fin de diagnosticar la situación económica de las industrias creativas; estos estudios han permitido establecer que este tipo de industrias se caracteriza por su dinamismo desde el punto de vista económico. 
El Ministerio de TIC (2011) revela que en Colombia, el aporte que ha hecho la actividad creativa al producto interno bruto (PIB) fue de 1,78\% en 2007, según el Departamento Administrativo Nacional de Estadística (DANE), siendo muy parecido a los sectores conocidos de la economía nacional: energía eléctrica, productos químicos, impuestos sobre productos e importaciones. De otra parte, su tasa de crecimiento se sostiene por encima de la del resto de la economía.

Otra cifra importante a mencionar en cuanto a aspectos económicos relacionados con la producción de contenidos digitales, es la inversión que se hace en el sector de las Tecnologías de la Información y Comunicación, en cuanto a porcentajes del PIB. Colombia, referente a inversión porcentual en TIC es inferior a otros países de la región (Argentina, Brasil, Chile, México, Uruguay y Venezuela), es similar a países como Azerbaijan, Bosnia Herzegovina, República Dominicana, Ecuador, Namibia, Perú, Serbia y Suráfrica y mucho menor a la inversión de países desarrollados como Australia, Bélgica, Canadá, Francia, Alemania, Holanda, Corea del Sur e Inglaterra. (Ministerio de TIC, 2011).

Existe una baja utilidad en los contenidos digitales debido a que su existencia es mínima a nivel local, porque no hay contenidos que manejen los mismos contextos del ciudadano común, es decir, no contienen información referente a sus vidas o negocios, por lo tanto, los mismos usuarios no ingresan a Internet ya que no hay afinidad entre lo que viven y lo que encuentran en la red. Gran parte del contenido digital que los usuarios colombianos de Internet consumen, está en el exterior. Según Alexa ${ }^{1}$, uno de los ranking más confiables de contenido en Internet, los diez sitios más populares visitados por los colombianos, corresponden a páginas extranjeras. (Restrepo, 2011).

Un caso exitoso del uso de tecnología en Colombia es la telefonía celular, en los años noventa el teléfono celular era un lujo, hoy en día, el 94\% de la población tiene un celular. El uso del celular ha permitido que otros servicios como Internet también se masifiquen (Ministerio de TIC, 2011).

La principal fortaleza en la producción de contenidos en este momento, es el apoyo que le está brindando el gobierno a través del Ministerio de TIC con la política de promoción de industria de contenidos digitales "Plan Vive Digital", ya que con ésta se busca masificar el uso de Internet y fortalecer al país como centro de producción de contenidos digitales, con miras a triplicar las ventas hacia el año 2014 en aplicaciones y contenidos.

Jorge Restrepo, gerente de promoción de contenidos digitales del Ministerio de TIC realizó una presentación (Restrepo, 2011) en la cual expone el término "El nuevo dorado", el cual hace alusión a un nuevo tesoro que es la creatividad colombiana en el campo del desarrollo digital. El Dr. Restrepo

\footnotetext{
${ }^{1}$ Alexa Internet es una subsidiaria de la compañía Amazon.com, conocida por operar el sitio web alexa.com que provee información acerca de la cantidad de visitas que recibe un sitio web dado. Alexa recoge información de los usuarios que tienen instalado Alexa Toolbar, lo cual le permite generar estadísticas acerca de la cantidad de visitas y de los enlaces relacionados.
}

Producción de contenidos digitales, una oportunidad para los profesionales de las TIC

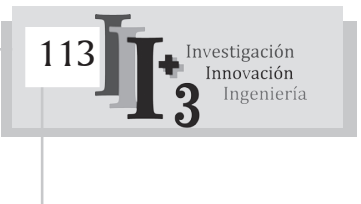


hace referencia a los contenidos digitales de: aplicaciones móviles, video juegos, animación digital, publicidad digital, video digital, libros digitales y bienes virtuales.

Entre las actividades a realizar por parte del Ministerio de TIC para apoyar la política de Contenidos Digitales, con miras a triplicar las ventas hacia 2014 están las siguientes:

- Realizar foros regionales con el sector privado y la academia

- Incentivar y facilitar el acceso a los contenidos digitales

- Financiar e incentivar la creación de empresas de contenidos: capital semilla e inversionistas.

La política de contenidos digitales incluye necesariamente un programa de capacitación en estos temas y en todos los niveles, empezando desde un plan de alfabetización digital para aquellos que no están familiarizados con el uso de la tecnología (manejo de internet y demanda de servicios), para esto se trabaja con programas del Ministerio de Educación Nacional - MEN, hasta la capacitación de profesionales, para lo cual se tienen proyectos con el Servicio Nacional de Aprendizaje (SENA) sobre formación en TIC y con Corea, en todo lo relacionado con producción de contenidos digitales, a través de pasantías de seis meses en ese país. Igualmente se desarrollarán programas de doctorado con Corea a través de un plan de cooperación internacional (Dinero.com, 2010).

\section{Cifras de Contenidos Digitales en Colombia}

Emprendedor (2011) presenta algunas cifras que son importantes de mencionar en cuanto a producción de contenidos digitales en Colombia:

- La facturación anual es de 70 millones USD por concepto de animación, 3D, contenidos en alta definición y video juegos, entre otros.

- El monto de las exportaciones asciende a los 10 millones de USD.

- Existen aproximadamente 7 series animadas para televisión, producidas en Colombia con mucho éxito, tales como: El siguiente programa, Betty Toons, El profesor súper O, El libro de Sofía, Los Pepa, Vulgarcito, La Familia del DT, entre otros.

\section{El Ecosistema Digital}

Este modelo fue desarrollado por el Banco Mundial para poder visualizar los elementos que permiten la "masificación del uso de Internet en una sociedad y sus interacciones" (Restrepo, 2011). Este modelo consta de cuatro componentes: infraestructura, servicios, aplicaciones y usuarios; además diseña estrategias para promoverlos. 
Como se puede observar en la Figura 2, los elementos del ecosistema están vinculados a la armonización de la oferta (infraestructura y servicios) y la demanda (usuarios y aplicaciones que contribuyen a generar demanda). (Orduz, 2010).

Para poder tener una realimentación positiva en el Ecosistema Digital, se necesita que exista una oferta y una demanda de servicios digitales, a través de aplicaciones y contenidos desarrollados en el país y que sean de interés para el mismo colombiano, utilizando Internet, permitiendo que el costo para el usuario final disminuya y generando una cadena que beneficie a las personas.

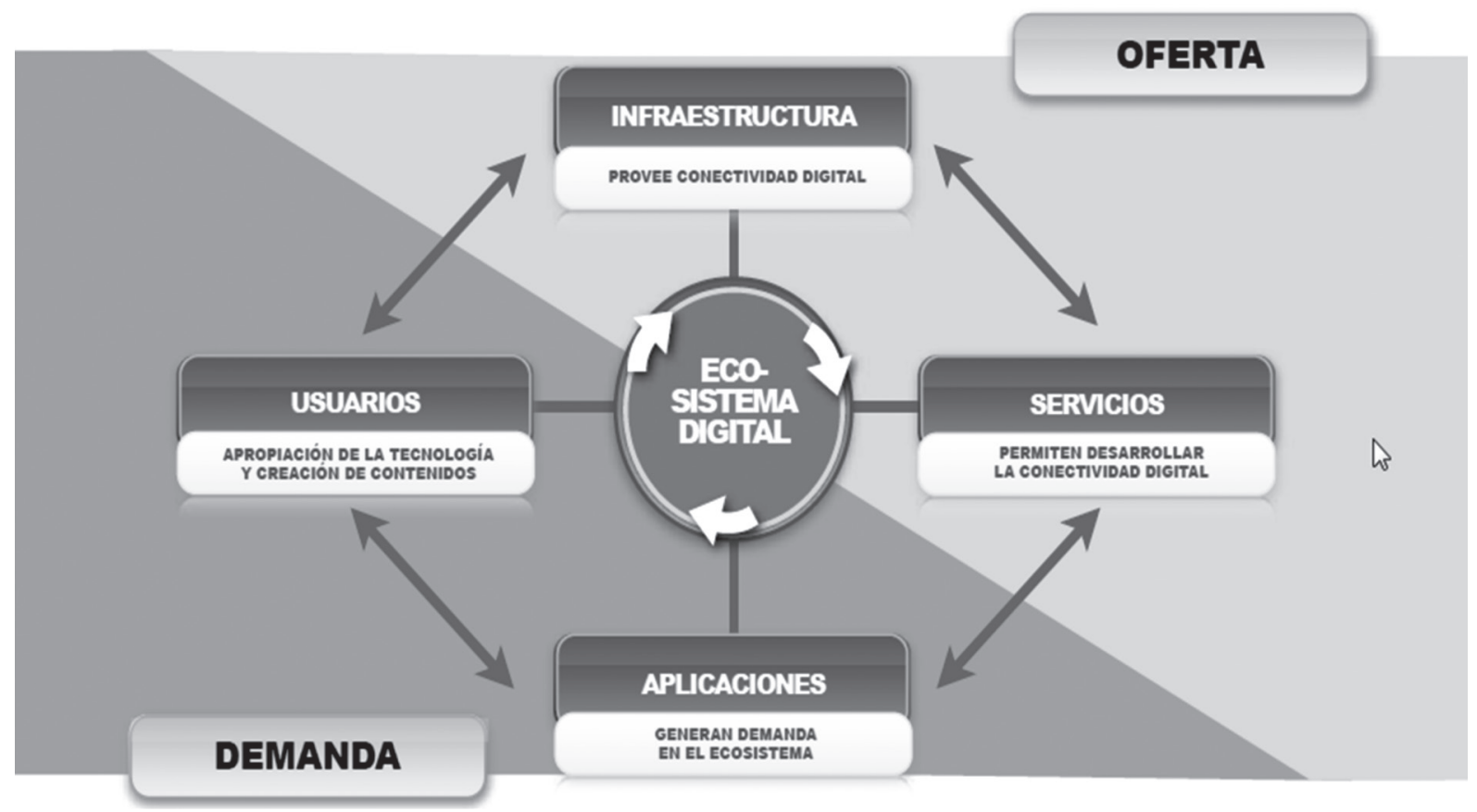

Figura 2. Ecosistema digital Ministerio de TIC . Fuente: Plan Vive Digital Colombia, 2011

El mapa mental que se muestra en la Figura 3, sintetiza los conceptos y metas propuestos en el Ecosistema Digital Colombiano. "El país cuenta con el talento humano suficiente para sobresalir en la región, esta política incentivará la oferta de bienes y servicios creativos y se estima que la facturación anual de la misma alcanzará los 748 millones de dólares anuales. Esto está enfocado al programa de transformación productiva y a la exportación de servicios de valor agregado como son los contenidos digitales", indicó el Ministro de Comercio, Industria y Turismo, Sergio Díaz-Granados (Briceño, 2011). 


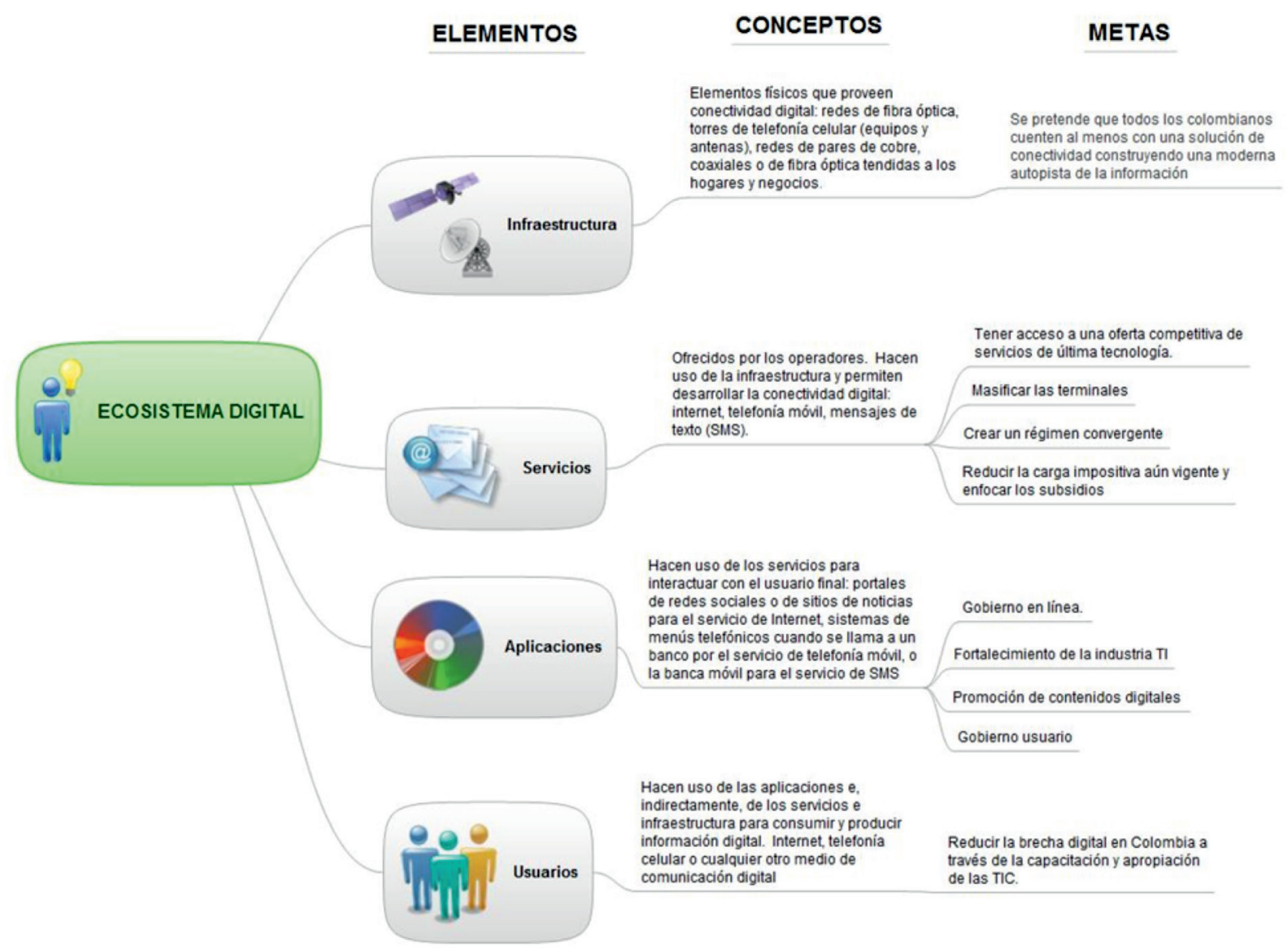

Figura 3. Ecosistema Digital Colombiano: Conceptos y metas. Fuente. Basado en Orduz, 2010 y Prieto Herrera, 2011

\section{Políticas públicas nacionales y marco legal}

El gobierno ha puesto en marcha una política, denominada Plan Vive Digital, que incentivará el uso de TIC, la cual incluye dentro de sus estrategias, la promoción de la industria de Contenidos Digitales.

El Plan Vive Digital es un Plan Estratégico Sectorial que busca masificar el uso de Internet y el desarrollo del ecosistema digital colombiano (mencionado en anteriores apartes) con miras a la apropiación y uso de las TIC. Se menciona en primera instancia el uso de Internet, puesto que al usar esta tecnología se incentivan otros espacios como la generación de aplicaciones y servicios para este mismo, obteniendo beneficios sociales y económicos. 
En la propuesta del Plan Vive Digital (Ministerio de TIC, 2011) se mencionan también las barreras encontradas para cumplir sus objetivos, haciendo el respectivo análisis y presentando estrategias para solventarlas. Entre ellas está el apoyo dado a la producción de contenidos digitales, a través del fortalecimiento de la asociatividad para impulsar el emprendimiento con TIC.

La política del plan vive digital también incluye el marco legal de la industria de contenidos digitales en Colombia, (Ministerio de TIC, 2011) dentro de las que se mencionan:

Ley 1341 de 2009, por la cual "se definen principios y conceptos sobre la sociedad de la información y la organización de las tecnologías de la información y las comunicaciones - TIC, se crea la Agencia Nacional del Espectro y se dictan otras disposiciones"

Propiedad intelectual y derechos de autor. Por el tipo de producto que se obtiene en la industria de contenidos digitales, es imprescindible tener en cuenta el marco legal referente a propiedad intelectual y derechos de autor. El centro de información de patentes de la Universidad Nacional (s.f.), presenta la siguiente normatividad en relación con este tema:

- Decisión 351 de la Comunidad Andina de Naciones (Régimen Común sobre Derecho de Autor y Derechos Conexos)

- Ley 48 de 1975 (adhesión a la Convención Universal sobre Derechos de Autor, sus Protocolos I y II y a la Convención Internacional sobre la Protección de los Artistas, Intérpretes o Ejecutantes, los Productores de Fonogramas y los Organismos de Radiodifusión)

- Ley 23 de 1982 (Sobre Derechos de Autor)

- Ley 33 de 1987 (adhesión al Convenio de Berna "Para la Protección de las Obras Literarias y Artísticas")

- Ley 44 de 1993 (modifica y adiciona la Ley 23 de 1982 y modifica la Ley 29 de 1944)

- Ley 545 de 1999 (adhesión al Tratado de la OMPI sobre interpretación o ejecución y fonogramas)

- Ley 565 de 2000 (adopción del Tratado de la OMPI sobre Derechos de Autor)

- Decreto 1360 de 1989 (inscripción del soporte lógico (software) en el Registro Nacional del Derecho de Autor)

- Decreto 460 de 1995 (reglamentación del Registro Nacional del Derecho de Autor y regulación del Depósito Legal)

- Decreto 162 de 1996 (reglamentación de la Decisión Andina 351 de 1993 y la Ley 44 de 1993, en relación con las Sociedades de Gestión Colectiva de Derecho de Autor o de Derechos Conexos)

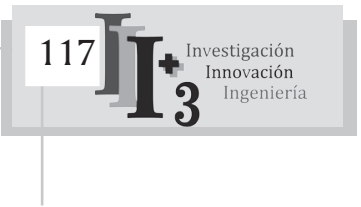


- Decreto 1070 de 2008 (por el cual se reglamenta el artículo 26 de la Ley 98 de 1993.)

- Ley de la protección de los datos personales. A la fecha está en estudio por parte del congreso de la república.

- Ley de delitos informáticos (1273 de 2009)

- Otras leyes relacionadas con la industria de contenidos digitales educativos: Ley del Libro (98 de 1993) y el Sistema Nacional de Cultura (397 de 1997).

\section{Promoción de la Industria de Contenidos Digitales}

La promoción de la industria de contenidos digitales en Colombia se materializó con el Plan Vive Digital (Ministerio de TIC, 2011) buscando con éste fomentar la industria de contenidos digitales en Colombia y promover la creación de aplicaciones y contenidos digitales. Con esta política, el gobierno busca apoyar a las personas que tienen formación y experiencia en esta área, para que generen empresas de producción de contenidos digitales y comercialicen sus productos en todo el mundo.

La política de promoción de Contenidos Digitales busca desarrollar y fortalecer la cadena de valor de la industria (creatividad, ideas, insumos, desarrollo de capacidad/preproducción, producción y acceso a mercados) de forma integral, fortaleciendo su talento humano, los mecanismos de financiación, la optimización de los procesos de producción, la promoción de la innovación y facilitar el acceso a mercados nacionales e internacionales.

"Este paso está alineado con las metas del Plan Vive Digital que busca triplicar los ingresos de contenidos digitales del país al 2014. Para el 2012 las proyecciones indican que esta industria alcanzará unos ingresos a nivel global de 2.200 billones de dólares, es decir, esta industria tiene un potencial mundial 28 veces mayor que la industria del café. Los ingresos del mercado mundial del café ascienden a 80 mil millones de dólares", señaló el Ministro TIC, Diego Molano Vega. (Briceño, 2011)

Dentro de las actividades planeadas en el plan Vive Digital, está la formación de cuatro laboratorios de creación de contenidos digitales llamados ViveLabs, los cuales permitirán trabajar de manera colaborativa, procesos que incluyen la formación técnica para incentivar el emprenderismo enfocado al tema. (Briceño, 2011)

\section{OPORTUNIDADES PRIORITARIAS PARA LA INDUSTRIA DE CONTENIDOS DIGITALES EN COLOMBIA}

Es procedente considerar el contexto de la Industria Colombiana de Contenidos Digitales, por lo que identificar las prioridades del sector se hace necesario. Los tiempos y ventanas de oportunidad de los 
contenidos digitales se consideran vitales y el grado de éxito potencial de los mismos depende de la innovación y la creatividad. (Latinpymes, 2011)

La creatividad en Colombia (Ministerio de TIC, 2011) es considerada de alta calidad, pero la capacidad para poner en circulación las producciones es limitada y obsoleta. A nivel global esta industria ha sufrido en los últimos años cambios constantes que obligan a las organizaciones del sector, tanto a innovar permanentemente los productos, como a actualizarlos, pues los avances de la tecnología y los cambios en los hábitos de consumo cambian constantemente.

En el primer foro nacional sobre contenidos digitales, realizado en la Universidad de Los Andes (Andes, 2011), Carlos Smith, representante de la Asociación Internacional de Films Animados (ASIFA), manifestó que hay personas que se han capacitado fuera del país en contenidos digitales y han constituido sus empresas, pero se encuentran desorganizados y no tienen una buena capacidad de producción. ASIFA tiene como objetivo fomentar el sector, para lo cual considera necesario vincular a docentes e investigadores con el fin de apoyar la creación de planes de estudio y desarrollar plataformas online para capacitación en animación digital.

Las empresas colombianas de animación digital carecen de procesos de comercialización estandarizados que les permitan llegar con éxito al mercado internacional, es por ello, que se requiere de tres elementos que se muestran en la Figura 4. 


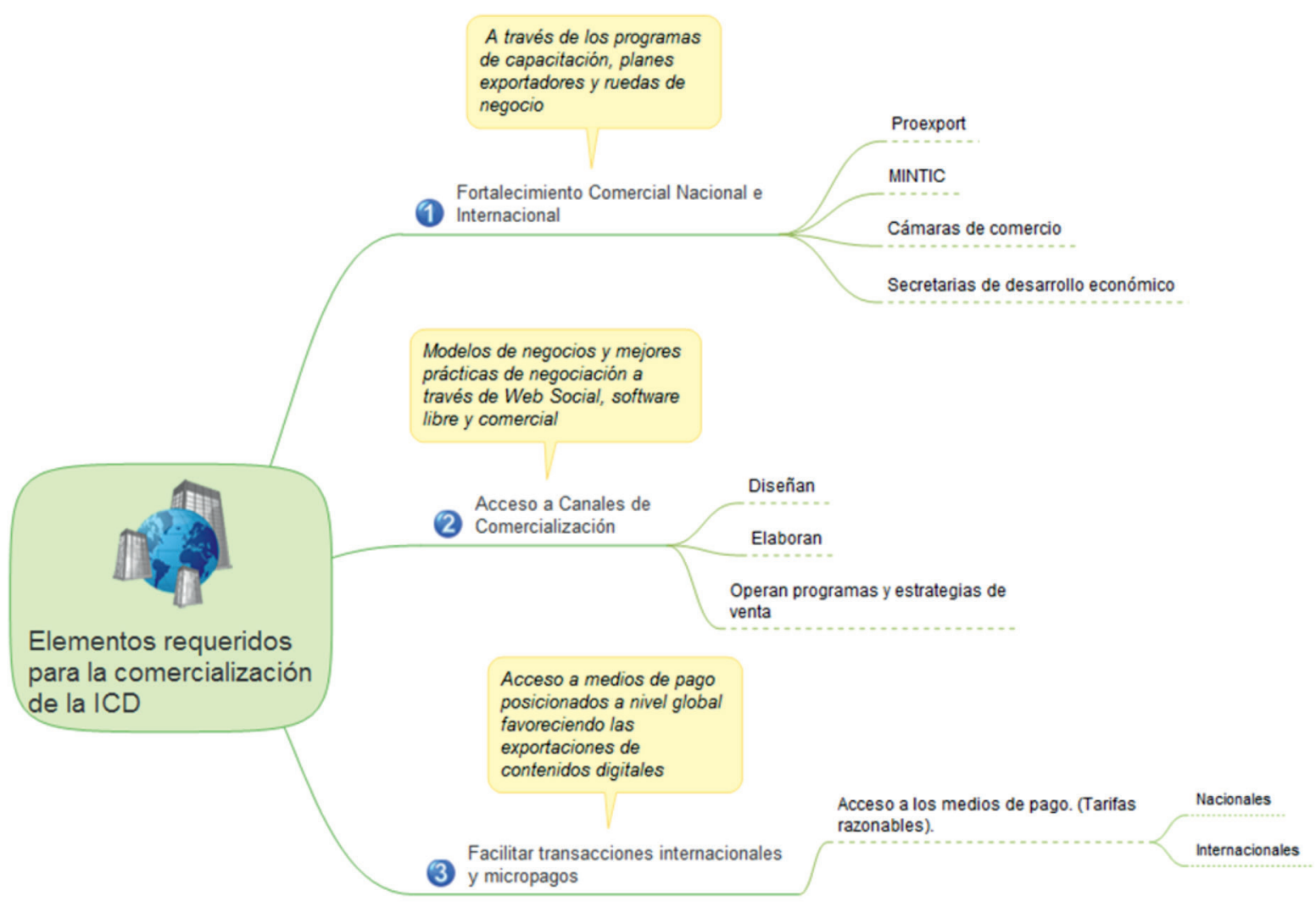

Figura 4. Elementos para la comercialización de la industria de contenidos digitales. Fuente: Basado en Ministerio de TIC, 2011 - PlanVive Digital Colombia.

La promoción y mecanismos de financiación para el desarrollo de la industria de contenidos digitales (ICD) son necesarios en el país y cuentan con una estructura de financiación especializada para el sector, con herramientas innovadoras y a la medida de la industria, considerando toda la cadena de inversión, desde capital semilla hasta fondos de capital de riesgo y mercado de capitales y soportando las necesidades de recursos económicos en los diferentes eslabones de la cadena de valor del sector (Apps, 2008-2012). Estos aspectos se muestran en la Figura 5. 


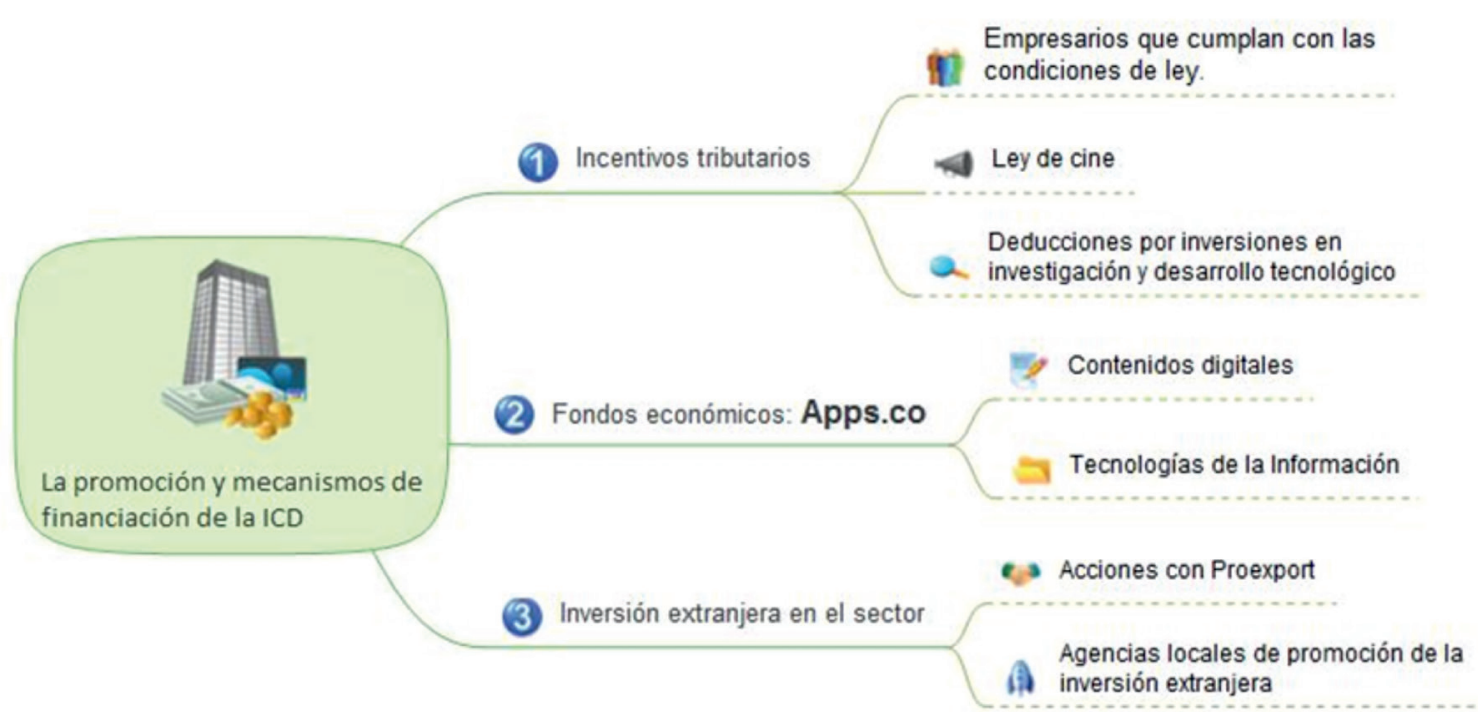

Figura 5. Promoción y mecanismos de financiación de la ICD en Colombia. Fuente. Basado en Ministerio de TIC, 2011 - Plan Vive Digital Colombia.

El desarrollo y difusión de la industria de contenidos digitales en Colombia se genera a través de acciones enfocadas a difundir el potencial de la industria, motivar el trabajo en red entre los empresarios, generar espacios de transferencia de conocimiento y compartir experiencias. En Colombia se difunde esta industria a través de varios eventos que se mencionan a continuación (Ministerio de TIC, 2011 - Plan Vive Digital):

- El evento Colombia 3.0 se proyecta como el gran evento anual del sector de las TIC y los contenidos digitales en el país, estará compuesto por actividades académicas como de negocio, ver página Web http://www.col30.co/index.php?lang=es. Contará con la participación de expertos y compradores internacionales. Se espera que tenga una gran convocatoria, debido a que se desarrollarán eventos regionales simultáneos usando la conectividad para multiplicar el impacto de este evento.

- Los foros sobre contenidos digitales tienen como objetivo difundir la política, sus acciones, las oportunidades del mercado y tendencias del sector, entre otros temas claves de la industria; así como mantener un diálogo permanente con los principales actores de la industria tales como: empresarios, académicos, emprendedores y estudiantes. 
Los eventos de promoción del emprendimiento en Contenidos Digitales son relativamente jóvenes en Colombia y están en constante cambio en el mundo entero, donde los modelos y prácticas de negocio evolucionan continuamente y se han alejado de los modelos tradicionales de emprendimiento y dirección de empresas.

En cuanto a oportunidades para la academia, las instituciones de educación superior pueden ofrecer programas de capacitación a través de cursos, programas de pregrado y postgrado, teniendo en cuenta las áreas del conocimiento que intervienen en el sector de los contenidos digitales (Interactic, 2011). A continuación se detallan cada una de ellas con el propósito de conocer la extensión que tienen:

- Creación de software: Software para dispositivos móviles, software en línea, software libre y software comercial.

- Diseño de contenidos digitales: Animación, objetos de aprendizaje, multimedia, juegos, música, videos, material didáctico (libros digitales, cartillas, e-learning), entre otros.

- Gerencia de producción de contenidos digitales: Plan de negocios, difusión, distribución, mercadeo y gestión de empresas de contenidos digitales.

\section{CONCLUSIONES}

Es conveniente analizar las iniciativas que otros países han tenido, respecto al desarrollo de la industria de contenidos digitales, para determinar si son aplicables en nuestro entorno e implementarlas con el fin de mejorar la economía creativa de nuestro país.

La industria de contenidos digitales brinda oportunidades para el emprendimiento en diferentes sectores como: Animación, diseño gráfico, multimedia, aplicaciones móviles, juegos, material didáctico digital, e-learning y educación en general.

Al masificarse Internet, se abren oportunidades para las personas que están involucradas en el área de las TIC, ya que con el ecosistema digital se ha empezado a generar un ciclo en donde será necesaria la implementación de infraestructura, servicios y aplicaciones.

Es importante conocer y aprovechar el apoyo que está brindando el gobierno Colombiano a través de organizaciones privadas y públicas, para generar nuevas empresas en el sector de los contenidos digitales y de las TIC.

Con el impulso que se está dando a la industria de contenidos digitales, la academia tiene grandes oportunidades para crear nuevos programas de formación en esta área e iniciar investigaciones relacionadas con esta temática.

Es necesario crear contenidos digitales que contengan temas que cubran intereses del ciudadano colombiano común, para que haya mayor acceso a internet y así abrir nuevos mercados. 


\section{REFERENCIAS BIBLIOGRÁFICAS}

Andes, U. d. (14 de Junio de 2011). Foros Isis. Recuperado el 15 de Septiembre de 2011, de http://sistemas.uniandes.edu.co/ foros/contenidos-digitales/memorias-primer-foro-de-contenidos-digitales/

APPS Ltda. (2008 - 2012). Application Development Team. Recuperado el 8 de Septiembre de 2011, de http://www.apps.com.co/apps/

Briceño, I. (22 de Junio de 2011). Radio Santa Fe . Recuperado el 12 de Septiembre de 2011, de http:// www.radiosantafe.com/2011/06/22/mintic-busca-fortalecer-la-industria-de-vontenidos-digitales/

Dinero.com. (28 de septiembre de 2010). [Entrevista con Patricia Asmar, asesora del Ministerio TIC en el componente de investigación, desarrollo e innovación y con Alejandro Delgado, asesor del Viceministerio de TIC en el tema contenidos digitales: Contenidos Digitales]. [Video en línea]. Consultado: [18, septiembre, 2011] Disponible en: http: http://www.ustream.tv/recorded/9872693\#utm_campaign=synclickback\&source $=$ http://alejandrodelgadomoreno.com/2010/09/ politica-de-contenidos-digitales-en.html\&medium $=9872693$.

Emprendedor, P. S. (23 de Junio de 2011). Soy emprendedor. Recuperado el 9 de Septiembre de 2011, de http:/www.soyemprendedor.net/index.php?option =com_k2\&view $=$ item\&id =1736: colombia-busca-consolidarse-como-centro-de-produccion-de-contenidos-digitales\&ltemid $=401$

Interactic, (Septiembre de 2010). Interacción con la información. Recuperado el 9 de Septiembre de 2011, de http:/www.interactic.org.co/noticintel/primer-plano/1933-foro-de-mintic-contenidos-digitales-convergentes-una-oportunidad-para-colombia

Latinpymes, (Septiembre de 2010). Centro de desarrollo empresarial. Recuperado el 12 de Septiembre de 2011, de http:/latinpymes.com/site/2011/06/en-colombia-los-contenidos-digitales-son-una-industria-con-futuro/

Ministerio de TIC. (Febrero de 2011). Vive digital Colombia. Recuperado el 13 de Septiembre de 2011, de http://www.vivedigital.gov.co

Orduz, R. (7 de Diciembre de 2010). Colombia Digital. Recuperado el 12 de Septiembre de 2011, de http:/www.colombiadigital.net/rafael-orduz/item/458-plan-vive-digital-colombia-ii.html

Prieto Herrera, A. (s.f.). Asociación nacional de anunciantes. Recuperado el 12 de Septiembre de 2011, de http://www.andacol.com/php/index.php?option $=$ com_content\&view $=$ article\&id $=334$ :lo-que-le-espera-a-colombia-con-las-tic\&catid $=46$ :revista-anda-44\&Itemid $=300117$

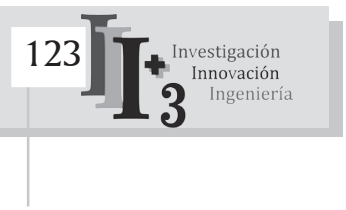


República, L. (22 de Junio de 2011). La República. Recuperado el 15 de Septiembre de 2011, dehttp:// www.larepublica.co/archivos/TECNOLOGIA/2011-06-22/gobierno-lanzo-politica-de-contenidos-digitales-para-estimular-la-inversion_131512.php

Restrepo, Jorge. (2011). Foro sobre contenidos Digitales. El nuevo Dorado. Universidad de Los Andes. Bogotá. [En línea]. Consultado: [4, julio, 2012] Disponible en: http://sistemas.uniandes.edu.co/ foros/contenidos-digitales/memorias-primer-foro-de-contenidos-digitales/

UNCTAD - UNITED NATIONS CONFERENCE ON TRADE AND DEVELOPMENT. (2010). Creative Economy Report. 2010. [En línea]. Consultado: [30, agosto, 2012] Disponible en: http://www.unctad.org/en/ docs/ditctab20103_en.pdf

UNIVERSIDAD NACIONAL DE COLOMBIA. (s.f.). Centro de información de patentes. Normatividad de propiedad intelectual. Normatividad relativa a Derecho de Autor. Recuperado el 31 de agosto de 2012, de: http://www.dib.unal.edu.co/cip/propiedadintelectual.html 


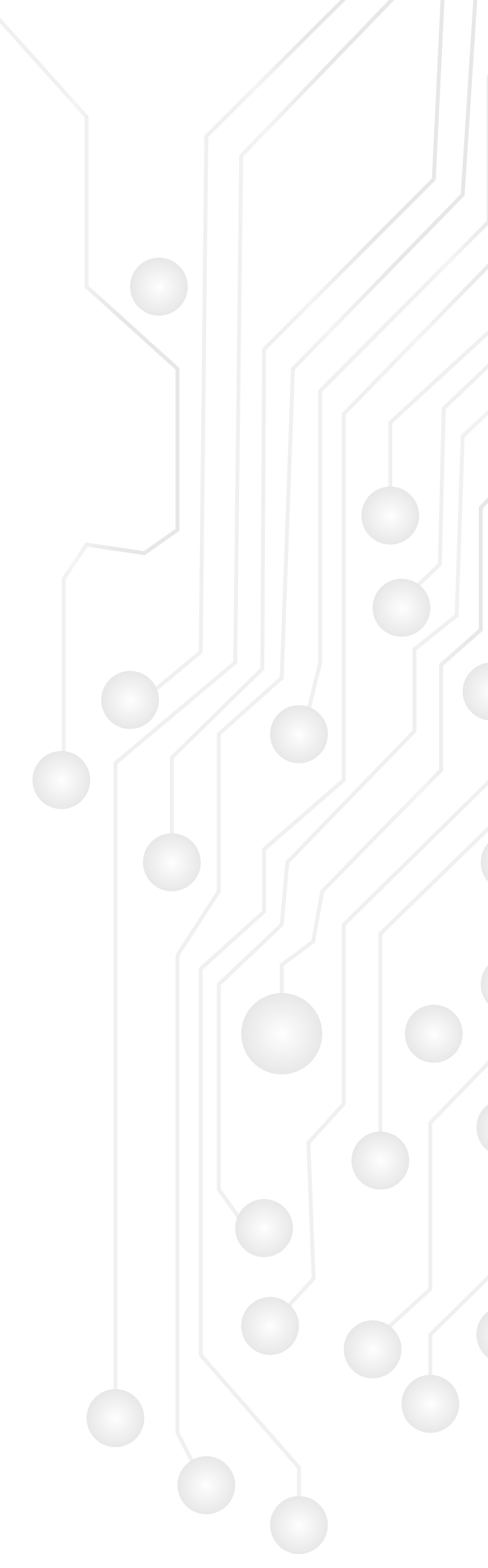

